\title{
Présentation
}

\section{Thomas Klinkert}

\section{(2) OpenEdition \\ 1 Journals}

Édition électronique

URL : https://journals.openedition.org/recherchestravaux/797

DOI : 10.4000/recherchestravaux.797

ISSN : 1969-6434

Éditeur

UGA Éditions/Université Grenoble Alpes

Édition imprimée

Date de publication : 1 juin 2016

Pagination : 5-12

ISBN : 978-2-84310-325-4

ISSN : 0151-1874

Référence électronique

Thomas Klinkert, «Présentation », Recherches \& Travaux [En ligne], 88 | 2016, mis en ligne le 01 janvier 2017, consulté le 29 octobre 2021. URL : http://journals.openedition.org/recherchestravaux/797 :

DOI : https://doi.org/10.4000/recherchestravaux.797 


\section{Présentation}

Depuis l'Antiquité, l'otium est considéré comme une forme d'expérience privilégiée qui s’oppose à la sphère de la vie quotidienne dominée par les devoirs et les affaires. Le terme latin otium est en opposition avec negotium, c'est-àdire «les affaires», "le devoir», en particulier ceux d'un homme d'État, d'un homme politique, de celui qui s'occupe de la res publica. C'est cette opposition qui structure les réflexions du philosophe stoïcien Sénèque contenues dans son texte De otio, où il dit que la nature a créé les hommes dans le but de s'adonner autant à la contemplation qu'à l'activité (De otio, 5, I). Dans ce texte, il dit aussi qu'il est préférable pour l'homme de se vouer à l'otium parce que c'est dans cet état que le sage peut chercher à comprendre les secrets du monde et de l'univers. L'otium est donc une attitude indispensable pour celui qui s'efforce d'arriver à la connaissance, telle que celle-ci a été conçue par la nature même, car elle a doué l'homme d'un esprit curieux et de la faculté de percevoir et d'admirer les

I. L'idée de réaliser ce numéro de Recherches \& Travaux est née en 2014 au sein du Centre de recherche collaborative (Sonderforschungsbereich), soutenu depuis 2013 par la Deutsche Forschungsgemeinschaft (DFG), situé à l'université de Fribourg-en-Brisgau et se consacrant à l'étude interdisciplinaire de l'otium: «Muße: Konzepte, Räume, Figuren». J'étais alors membre de cette université et faisais également partie du SFB. Malgré mon départ pour l'université de Zurich en 2015, je suis toujours membre du SFB et tiens à remercier tous les collègues de Fribourg et du SFB avec qui j'ai eu le plaisir de réfléchir sur les différents aspects de l'otium et dont plusieurs ont écrit une contribution à ce volume (par ordre alphabétique : Georg Feitscher, Robert Krause, Heidi Liedke, Dieter Martin, Anna Karina Sennefelder), ainsi que tous les autres contributeurs venant d'universités situées en Allemagne, en Suisse, en France et au Canada, ce qui montre bien la dimension internationale de ce sujet de recherche. Je tiens à remercier en outre Cécile Roche, qui a traduit en français les articles de Georg Feitscher et de Dieter Martin, Anna Pevoski et Clara Schwarze, qui ont traduit celui de Heidi Liedke et m’ont assisté lors de la préparation de ce dossier, ainsi que Fabiola Valeri et Silvia Riccardi. Je remercie également Claude Coste et Bertrand Vibert pour m’avoir invité à éditer ce numéro. 
beautés de la création. Le sage qui contemple les beautés de la nature afin de les comprendre est comme un spectateur dans le théâtre du monde. En prenant volontairement la décision de se retirer du monde des affaires et de la res publica, le sage, en se donnant la possibilité de pénétrer les secrets de la nature, suit la destinée de l'homme.

Tout en se retirant du monde, cependant, il rend service à la société puisque le savoir qu'il acquiert dans la solitude de l'otium et de la contemplation peut être communiqué aux autres - grâce aux livres. L'otium est donc une qualité essentielle de la condition humaine, ce qui signifie que pour réaliser pleinement les capacités et les facultés qui lui ont été données par la nature, l'homme a besoin de l'otium. Celui-ci est un des facteurs les plus importants qui définissent l'humanité de l'homme et, par conséquent, la félicité humaine, que les Grecs appelaient eudaimonia. En même temps, l'otium est en rapport étroit avec la communication, si bien qu'il semble légitime de s'interroger sur la relation entre otium et écriture.

Dans cette perspective, on peut constater que l'otium a souvent servi comme point de départ d'une activité littéraire. Par exemple, dans Le Décaméron de Boccace (vers I350) il y a une opposition fondamentale entre la ville de Florence menacée et détruite par la peste et la sphère de l'otium qui est située à la campagne et dans laquelle se retirent les dix jeunes protagonistes de ce livre. Une fois arrivés dans la sphère du locus amoenus, ils se consacrent à des activités de loisir (danse, musique, jeux); surtout, ils passent leur temps à se raconter des histoires. Ainsi, les membres de cette collectivité se construisent, avec les moyens de l'imagination littéraire, un monde qui repose sur des principes d'ordre, constituant une contrepartie au monde dévasté et désordonné qu'ils viennent de quitter. Le fait de se retirer dans un monde agréable est en outre justifié par des conceptions médicales de l'époque selon lesquelles l'air salubre de la campagne peut avoir une valeur thérapeutique, si bien que la situation d'otium des jeunes gens est un antidote contre la maladie contagieuse qu' est la peste $^{2}$. De façon comparable, l'otium peut servir de cadre à une entreprise de renoncement au monde telle que la réalise Michel de Montaigne à la fin du $\mathrm{XVI}^{\mathrm{e}}$ siècle. Après avoir vendu son poste de conseiller au parlement de Bordeaux, il se retire dans la tour de son château où il a fait aménager une bibliothèque. C'est là qu'il écrit ses Essais tout en jouissant de la présence des livres qui lui permettent d'entrer en contact dialogique avec les grands esprits du passé.

2. Voir G. Olson, Literature as Recreation in the Later Middle Ages, Ithaca, Cornell University Press, I982, p. I82. 
Dans ces deux cas exemplaires, l'otium peut être considéré comme une liberté qui permet à un groupe ou à un individu d'échapper à des contraintes ou à des dangers de la vie de leur temps pour se mettre dans un état de réflexion, de méditation ou de communication amicale. Dans cet état de liberté peut naître une créativité littéraire. En même temps, le fait de se retirer dans une situation d'otium est une protection contre les malheurs du monde. On peut donc formuler l'hypothèse que l'otium est un principe élémentaire de protection et de restitution qui permet aux êtres humains de maintenir leur intégrité corporelle et mentale dans des situations de menace 3 .

Cette tradition va être profondément ébranlée et bouleversée par le choc de la Révolution française et par la redéfinition du système culturel et social qui en découle. C'est ce que les contributions réunies dans ce volume se proposent d'étudier. Ainsi Franziska Meier entre-t-elle dans le vif du sujet de la Révolution en montrant que celle-ci fut perçue par les contemporains comme une catastrophe naturelle ou comme un torrent qui, en sortant de son lit, emporte tout sur son passage. Un des témoins de la Révolution, Rétif de la Bretonne observe, dans Les Nuits de Paris, le mouvement irrésistible de la foule et du peuple révolté dont il compare l'effet à une "décharge électrique" (infra, p. 17). Le tumulte des événements révolutionnaires entraîne l'observateur qui ne peut pas résister au mouvement de la foule; en même temps, ce tumulte s'empare de la forme du texte, dans la mesure où celui-ci est marqué par une "langue délirante» (infra, p. I8). Étant donné le caractère tumultueux et mouvementé des événements de la Révolution, on peut se demander à juste titre s'il est encore possible que des individus dans ces circonstances aient recours à l'otium. Or, en étudiant les ouvres de deux mémorialistes de l'époque, à savoir Marmontel et Madame Roland, Meier montre que d'une part, des éléments de la tradition de l'otium survivent, mais que d'autre part, la relation traditionnelle entre otium et écriture est bouleversée. Ce n'est plus l'otium qui donne lieu à l'écriture, mais c'est au contraire l'écriture "qui crée en quelque sorte pour l'écrivain une sensation d'otium [...], dans la narration d'un passé révolu et nettement séparé de la condition présente de l'auteur» (ibid., p. 27). De façon générale, les mémorialistes de la Révolution constatent que la corrélation traditionnelle entre écriture et otium est remise en cause par la nouvelle manière de vivre l'Histoire, "perçue comme un mouvement qui se déroule selon ses propres règles» (infra, p. 29).

3. Ces réflexions portant sur le rapport entre otium et écriture sont approfondies dans mon livre Muße und Erzählen - ein poetologischer Zusammenhang. Vom "Roman de la Rose» bis zu Jorge Semprún, Tübingen, Mohr Siebeck (à paraître en 20I6). 
Certains écrivains penseurs du XIX ${ }^{\mathrm{e}}$ siècle, en particulier Leopardi, Stendhal et Baudelaire, réagissent dans leurs œuvres à la situation postrévolutionnaire en essayant de développer une nouvelle esthétique adaptée aux bouleversements causés par l'événement que fut la Révolution tout au long du XIX ${ }^{\mathrm{e}}$ siècle. C'est en réfléchissant sur la nouvelle manière de vivre l'Histoire que ces écrivains définissent une esthétique de la modernité dans laquelle l'otium trouve une nouvelle place. Comme le montre Cosetta Veronese, chez Leopardi la notion d'otium se situe entre l'oisiveté et la noia (l'ennui). En analysant un texte des Canti, à savoir l'épître Au comte Carlo Pepoli, et en reconstruisant la pensée philosophique de Leopardi à travers des passages empruntés au Zibaldone et aux Petites CEuvres morales, l'auteur montre que pour Leopardi l'expérience de la noia s'associe à la perception du néant et que c'est dans cette association que se dessine "un profil ou un portrait du sujet moderne» (infra, p. 35). La pensée léopardienne conçoit l'Histoire comme un processus caractérisé par la perte de toutes les illusions de bonheur et la découverte irréversible du néant de l'existence par les humains. C'est la philosophie qui oblige l'homme moderne à s'apercevoir de l'insignifiance de l'existence, sans pour autant lui donner les moyens de combler ce vide. Si cette conscience du vide et du néant s'introduit également dans la poésie, créant ainsi une assimilation partielle entre poésie et philosophie et détruisant les illusions qui seules peuvent rendre les hommes heureux, une issue possible de cette impasse réside dans le domaine esthétique et dans ses ambivalences. Ainsi, comme le montre Cosetta Veronese, Leopardi développe une conception de l'esthétique reposant sur les idées d'inutilité, d'absence de but et de "légèreté dans le sérieux» (infra, p. 47). Cette conception va dans le sens de la «flânerie» telle qu'elle sera plus tard développée par Baudelaire et Benjamin.

Chez Stendhal, qui est, lui aussi, un penseur du nouveau rapport de l'homme à l'Histoire, le projet autobiographique, qui depuis Montaigne et Rousseau est associé à l'otium, est sujet à une reconfiguration significative, ainsi que le montre la contribution d'Anna Karina Sennefelder. Après avoir retracé la relation traditionnelle entre l'idylle, l'otium et l'introspection, elle montre le caractère novateur de l'entreprise autobiographique de Stendhal. Au début de la Vie de Henry Brulard, Stendhal crée une association forte entre un lieu hautement chargé de mémoires culturelles, à savoir le mont Janicule de Rome, et sa décision individuelle d'écrire l'histoire de sa vie. Or la datation explicite contenue dans le texte ne correspond pas à la genèse véritable de celui-ci, ce qui signifie que cette datation est l'expression d'une stratégie de stylisation employée par Stendhal. C'est en mettant en opposition «la pérennité de la ville et de l'art qui y subsiste depuis des siècles» et "le caractère éphémère du sujet» (infra, p. 6o) qui va bientôt avoir cinquante ans, que Stendhal motive 
son projet autobiographique, mais celui-ci ne pourra commencer véritablement que plus tard et ailleurs. En fait, le premier acte d'écriture véritable de cette autobiographie se produit en un lieu situé près du lac d'Albano dont la mémoire culturelle n'a rien à voir avec la richesse du mont Janicule. Le fait que ce lieu anodin soit libre de mémoire culturelle permet au sujet de l'autobiographie de se l'approprier grâce à l'otium et de se soustraire ainsi au mouvement de l'Histoire en s'inscrivant dans un texte littéraire.

Le troisième poète-penseur de la modernité et de l'Histoire traité dans ce volume est Charles Baudelaire. Dans son esthétique de la modernité, on le sait bien, c'est le flâneur qui incarne l'otium. Or, selon l'hypothèse de Diemo Landgraf, il y a chez Baudelaire une réévaluation de l'otium qui, comme chez Leopardi d'ailleurs, se teinte de négativité, car il se rapproche des notions de décadence et d'ennui. Cela s'explique, d'une part, par le fait que Baudelaire, dans sa tentative désespérée et vouée à l'échec de trouver un sens métaphysique à la vie, se voit réduit au nihilisme et d'autre part, par le fait qu'il refuse radicalement la société bourgeoise dans laquelle il vit. Par conséquent, la liberté de l'artiste dont il se réclame et qu'il cherche à réaliser dans l'otium de la flânerie ou de la production poétique s'avère une expérience décevante dans laquelle le sujet est en proie à l'ennui et au dégoût. C'est en étudiant quelques poèmes des Fleurs du Mal ("Au lecteur», "Bénédiction" et "Spleen» I et II) que Diemo Landgraf montre quelques-unes des réalisations esthétiques de la philosophie de Baudelaire, en tenant compte de la tension fondamentale qui caractérise l'œuvre de ce poète pris entre la tendance vers l'idéal et la souffrance causée par le spleen.

Si chez Leopardi, Stendhal et Baudelaire, l'otium subit des transformations et des redéfinitions qui s'expliquent par la pensée de l'Histoire caractéristique de ces auteurs, chez George Sand, l'otium se trouve mis en rapport avec la liberté et la création artistique d'une manière plutôt traditionnelle. Dans sa contribution, Gérard Peylet analyse trois romans d'artistes de George Sand (Teverino, Consuelo et Le Château des Désertes), montrant comment chez cet auteur les temps de rupture et de recul, le suspens de l'activité, sont mis à contribution pour permettre la création artistique. Cette création peut s'insérer dans une "poétique de la mobilité» (infra, p. 88) comme dans Teverino, qui raconte l'histoire de l'aristocrate Sabina et du dessinateur Léonce, qui sont des figures du franchissement des frontières, ou bien dans Consuelo où la rencontre de l'héroïne éponyme avec le jeune compositeur Joseph Haydn donne lieu à un voyage qui "favorise la révélation artistique» (infra, p. 92). Elle peut également se réaliser lorsque Consuelo se trouve en prison dans la mesure où cette expérience «libère le génie et la créativité de l'artiste» (infra, p. 94). Dans le Château des Désertes, enfin, roman qui fut écrit après la désillusion 
politique de i848, George Sand analyse «le double lien qui unit l'acteur à l'art et à la vie», racontant l'histoire d'un apprentissage théâtral sous forme d'une «expérience unique» (infra, p. 95), une "rénovation artistique» (infra, p. 99) qui se produit dans un lieu clos, à savoir un château, et dont le principe est l'art de l'improvisation.

Si dans ce dernier exemple de George Sand l'otium est mis au service d'une expérimentation artistique, chez William Henry Hudson, écrivain de voyage et naturaliste d'origine anglaise qui a passé les trente premières années de sa vie en Amérique latine, on peut constater un emploi systématique de l'état d'oisiveté dans le but d'une mise en marche de l'écriture, comme l'analyse Heidi Liedke dans sa contribution. Il s'agit d'une écriture artistique dont l'impulsion première est la solitude radicale que Hudson a connue durant ses voyages à travers la Patagonie, dont il parle dans son livre intitulé Un flâneur en Patagonie. L'oisiveté du voyageur patagon, qui est en partie une oisiveté forcée car elle fut d'abord provoquée par une blessure au genou, s'inscrit en faux contre les idéaux affairés de la société victorienne. Mais peu à peu, Hudson renonce à vouloir donner une justification extérieure à son oisiveté, et il se contente simplement de raconter comment il a progressivement adopté l'état d'esprit d'un oisif. C'est en cherchant volontairement l'isolement en Patagonie qu'il réussit à "s'approprier l'espace étranger» et à le «transformer en un refuge» (infra, p. II3). L'écriture cherche à rendre avec des moyens poétiques les impressions "épiphaniques" (infra, p. I20) du voyageur oisif, qui est volontairement entré dans la sphère du silence.

$\mathrm{Au} \mathrm{xx}^{\mathrm{e}}$ siècle, des auteurs comme Walter Benjamin reviennent sur l'esthétique de la modernité telle qu'elle avait été développée par Baudelaire au $\mathrm{XIX}^{\mathrm{e}}$ siècle. Ainsi que le montre l'étude de Robert Krause, Benjamin, dans sa nouvelle Myslowitz - Braunschweig-Marseille, explore ce que Baudelaire appelle les "paradis artificiels", à savoir l'ivresse provoquée par la consommation de drogues, et son rapport à la perception esthétique. L'otium joue un rôle central dans la nouvelle de Benjamin, qui consiste en un récit cadre et un récit enchâssé. Dans le récit cadre est évoquée la situation traditionnelle de l'otium collectif donnant lieu à une narration : des amis sont réunis autour d'une table dans un restaurant et se racontent des histoires. Or, dans le récit enchâssé, dont le narrateur est le peintre Eduard Scherlinger, plusieurs formes d'otium entrent en jeu : le narrateur-protagoniste part en voyage, laissant derrière lui le monde des affaires; il se rend dans la ville de Marseille, lieu de la flânerie; il y consomme du hachisch, ce qui le met dans un état d'otium très spécial. Ces diverses formes d'otium contribuent à la réflexion du sujet sur lui-même, sur son identité et sur la narration. Ainsi, l'ivresse provoquée par le hachisch se révèle notamment une «condition de possibilité de la constitution du sujet» (infra, p. 139). 
À côté de cette constitution «artificielle» du sujet moyennant l'expérience de la drogue, il y a également au $\mathrm{xx}^{\mathrm{e}}$ siècle une mise en cause radicale du sujet, représentée notamment par Robert Musil. En analysant le cycle narratif Le Merle et des passages choisis de L'Homme sans qualités, Dieter Martin montre la façon dont Musil met en scène dans ses textes narratifs ce que l'on peut appeler, avec une formule d'Ernst Mach, le "moi irrémédiablement perdu» (infra, p. I4I). À la différence de l'idée optimiste, défendue par le philosophe herméneute Wilhelm Dilthey, d'une constitution du sujet par les moyens de la narration autobiographique, selon Musil, il est impossible à un sujet qui se penche sur son propre passé dans une situation d'otium de jamais atteindre l'unité et le sens de sa propre vie en produisant une synthèse narrative. L'un des personnages de Merle prend ses distances par rapport à la volonté de créer une telle synthèse en utilisant la formule ironiquement dépréciative du «système de la Caisse d'épargne du moi» (infra, p. I4I). Chez Musil, ce renoncement volontaire à la synthèse autobiographique est contrebalancé par un emploi insolite de la forme narrative. Ainsi, au lieu de «donner un cours linéaire et épique au déroulement de leur vie, et de le raconter conformément au modèle autobiographique du fll», les deux personnages de Merle "entretissent les souvenirs, qu'ils invoquent par association d'idées, en une surface» (infra, p. 155).

Vers la fin du $\mathrm{Xx}^{\mathrm{e}}$ siècle, à l'ère postmoderne, on constate que les auteurs ont tendance à employer librement les topö̈ de la constitution narrative du sujet que la tradition met à leur disposition, créant ainsi des "postfigurations" du loisir. Ce phénomène est étudié par Georg Feitscher qui s’appuie sur deux exemples : la promenade et la randonnée en montagne. En comparant deux textes autobiographiques contemporains, à savoir Montauk de Max Frisch et Die weisse Einsamkeit. Mein langer Weg zum Nanga Parbat ${ }^{4}$ de l'alpiniste Reinhold Messner, avec deux modèles «classiques», soit Les Rêveries du promeneur solitaire de Rousseau et L'Ascension du Mont Ventoux de Pétrarque, Feitscher montre la persistance volontaire ou involontaire de certains topö̈. En réactualisant «plusieurs motifs rousseauistes, comme la fuite de la ville vers la nature, la promenade et le temps d'arrêt au bord de l'eau" (infra, p. 167), Frisch crée une postfiguration de l'autobiographie qui lui permet de réfléchir sur son passé d'homme et d'écrivain engagé et d'inaugurer une nouvelle poétique au sein de son œuvre. Quant à Messner, il réactualise "le topos du sommet de montagne, lieu de rencontre du monde et du moi» (infra, p. 172). Il est frappant de relever certains parallèles entre Pétrarque, Rousseau et Messner,

4. Soit en français : «La solitude blanche. Mon long chemin vers le Nanga Parbat». L'œuvre a en fait été traduite sous le titre Nanga Parbat, Paris, Arthaud, 20 Io. 
notamment en ce qui concerne «l'absence de but imposé, la marche solitaire et le monologue» (infra, p. I73).

Ainsi, les contributions de ce volume montrent dans une perspective diachronique comment, depuis la Révolution française jusqu’à la fin $\mathrm{du} \mathrm{Xx}^{\mathrm{e}}$ siècle, le rapport entre l'otium et l'écriture permet de définir la situation de la littérature par rapport à l'Histoire. C'est la Révolution qui, pour la première fois, confronte les individus de façon immédiate et radicale à l'accélération de l'Histoire et les entraîne dans un mouvement irrésistible. Ce nouveau rapport à l'Histoire a pour conséquence une redéfinition de l'otium. Ainsi, dans les conceptions esthétiques et poétologiques d'auteurs comme Leopardi, Stendhal et Baudelaire, l'otium est au cour de la nouvelle perception de l'Histoire. Si chez des écrivains comme George Sand ou William Henry Hudson, l'otium peut encore servir comme moment de retraite et de suspens donnant lieu à une activité artistique, cette fonction traditionnelle devient de plus en plus fragile. Au Xx siècle, l'otium peut être associé aux "paradis artificiels" causés par l'ivresse du hachisch comme chez Walter Benjamin, ou bien il est évoqué dans le cadre d'une mise en cause radicale de la constitution narrative du sujet comme chez Musil. À l'ère postmoderne, les vestiges de la tradition sont réactualisés sous forme de "postfigurations» du loisir. Dans tous les cas étudiés dans ce volume, l'otium permet de mesurer la portée historique et poétologique des textes et de faire ressortir le rapport qui réunit le texte littéraire, le sujet de l'énonciation et l'Histoire. 